\title{
Éléments ethnographiques
}

Les rites funéraires chez les Minyanka du Mali

From fieldwork: Funeral rites among the Minyanka of Mali

Danielle Jonckers

\section{(2) OpenEdition}

Journals

Édition électronique

URL : http://journals.openedition.org/span/126

DOI : 10.4000/span.126

ISSN : 2268-1558

Éditeur

École pratique des hautes études. Sciences humaines

Édition imprimée

Date de publication : 1 septembre 1975

Pagination : $35-51$

ISSN : 0294-7080

Référence électronique

Danielle Jonckers, «Éléments ethnographiques », Systèmes de pensée en Afrique noire [En ligne],

1 | 1975, mis en ligne le 02 juillet 2013, consulté le 19 avril 2019. URL : http://journals.openedition.org/ span/126; DOI : 10.4000/span.126 
ELEMENTS ETHNOGRAPHIQUES :

LES RITES FUNERAIRES CHEZ LES MINYANKA DU MALI

par Danielle JONCKERS

PROPOS : Cet article est le résultat de premières enquêtes ethnographiques ; une analyse complémentaire sera menée ultérieurement afin de dégager la signification profonde des rites observés et leurs implications au niveau économique et social.

La vie religieuse minyanka est très complexe, elle met en jeu non seulement un dieu créateur $k l \varepsilon$ et des puissances intermédiaires entre $k l$ et les hommes mais également les morts.

Notre propos, dans cet article, est de décrire les rites funéraires normaux occasionnés par le décès d'un vieillard et les rites particuliers liés au statut du défunt ou aux circonstances de sa mort. Il nous a paru intéressant de signaler ici le simulacre de funérailles qui se déroule lors de l'intronisation du chef de village, dans le nord du Minyankala.

\section{RESUME}

Les ancêtres sont invoqués et honorés par des sacrifices au niveau villageois et familial. Pour élever le défunt au rang d'ancêtre et pour éviter que les éléments de la personne, dissociés par le décès, ne viennent troubler les vivants, ceux-ci doivent accomplir les rites funéraires. Différents éléments du rituel nous indiquent que les Minyanka considèrent la mort comme une étape avant la résurrection. Tout le patrilignage et toutes les familles alliées doivent être présents aux funérailles et supporter les dépenses ostentatoires (repas, griots, sacrifices). Le corps avant d'être inhumé subit ceux toilettes et des onctions. La tombe sera différente selon le statut du défunt et les moyens de sa famille. Le cortège funèbre marque plusieurs arrêts avant le cimetière : des rites particuliers se déroulent à la porte de la concession lignagère et au cimetière des enfants. Les petits-enfants du mort jouent un rôle particulier : ils troublent le cortège funèbre et insultent le mort. Le veuf(ve) doit respecter certains interdits. Les cérémoniès funèbres se clôturent par un ou plusieurs repas rituels sans lesquels le défunt ne peut rejoindre l'au-delà. 
Les rites sont différents lorsqu'il s'agit de morts violentes ou de catégories particulières de personnes (femmes stériles, albinos) leurs dépouilles doivent être traftées avec prêcaution car leur contact avec la terre mettrait en jeu sa fertilité. Dans la partie nord du Minyankala, un simulacre de funérailles fonde la sacralité du pouvoir du chef de village.

L'intronisation se déroule en deux temps : lors de la première cérémonie le chef est coiffé d'un bonnet, insigne de son pouvoir. Après une réclusion de quarante deux jours, mort symbolique, le chef est présenté avec faste au public.

La communication avec l'au-delà occupe une place importante dans les activités rituelles. Dans leurs prières adressées aux esprits des morts, les hommes demandent santé, prospérité et féconđité, éléments essentiels à la vie dont les déterminismes échappent en tout ou en partie à leur emprise technique. Les vivants pensent que les ancêtres (kule) peuvent leur accorder ces bienfaits et ils se sentent moralement obligés de se soumettre à leur autorité et de leur faire des offrandes. Chaque année, avant les semailles et après les récoltes, le chef de village invoque les ancêtres fondateurs : il effectue des sacrifices au nom de la collectivité au bois sacré (kachi kã) et à la porte monumentale du village kunyu gbo (porte, grande).

Au niveau familial, la grande cuisine où le patriarche offre les prémices des récoltes-et la grande porte de la concession où il procède aux libations et aux sacrifices sanglants- sont le theatre du culte des morts.

La grande cuisine est la cuisine du lignage, souvent désaffec- . tée suite à l'éclatement des familles. Elle reste néanmoins un lieu de culte et rappelle l'unité du patrilignage.

La grande porte témoigne également de cette unité. Tout changement de statut important (mariage, décès, exclusion) dans la vie d'un membre du lignage est marqué par le passage de cette porte. 
Pour elever le defunt au rang d'ancêtre et lui garantir son statut au village des morts $y \tilde{\alpha} k \tilde{\alpha}$, (mort, village), les vivants doivent accomplir les rites funéraires. La mort $(k u)$ dissocie les éléments composant la personne : le nyama véhiculé par le sang, le souffle (muna) (1) et l'ombre, le double dya.

Ces forces spirituelles quittent le corps du mort, et le dya peut se manifester tant que les funérailles ne sont pas terminées ou si le culte des ancêtres est négligé.

Les proches assistent aux derniers moments du mourant; son fils ainé doit lui donner à boire avant qu'il n'expire sans quoi le dya reviendra.

Un frère, un fils ou un ami du défunt se rend chez le devin pour connâ̂tre les causes de la mort, qui déterminent les rites funéraires.

Des coups de fusil sont tirés à blanc, pour signaler le décès et des émissaires sont envoyés pour prévenir les parents éloignés. Tout le patrilignage, $y$ compris les filles mariées, et les familles alliées doivent être présents aux funérailles

Les dépenses ostentatoires occasionnées par les funérailles, (et qui peuvent représenter de 1000 à $5000 \mathrm{FF}$ ), sont supportées par les fils du défunt et par les chefs des familles alliées ou sont mariées les filles du défunt. Les fils procurent les animaux de sacrifice, les denrées pour les repas et paient les chanteurs et les musiciens.

Chaque fille mariée se présente aux funérailles accompagnée d'un griot (2) et offre 5000 à 15000 cauris (3), une natte et des pagnes rayés noir rouge et blanc ou des couvertures blanches. La fille ainée apporte la tenue funéraire : un grand boubou ou un pagne blanc. Tous les participants donnent de l'argent et des cauris au chef de Eamille du défunt ainsi qu'aux chanteurs et musiciens.

(1) Ce terme désigne également le nez, l'expression "couper le nez" signifie tuer quelqu'un.

(2) Chez les Minyanka, les griots ne constituent pas une caste dans le sens d'une catégorie socio-professionnelle endogame.

(3) 2500 cauris : $4 \mathrm{gr} 238$ d'or, dans Majhemout Diop, Histoire des classes sociales dans l'Afrique de l'Ouest, tome 1. Le Mali. Paris, F. Maspéro, 1971, Les textes à l'appui. 
Les biens remis au chef de famille sont largement distribués aux griots et aux petits-enfants du défunt à qui il faut "acheter le mort".

Les grands-parents et les petits-enfants entretiennent entre eux des rapports quasi égalitaires, ils échangent des plaisanteries dont le thème favori est leur mariage, ils "s'appartiennent" mutuellement.

Aussitôt la mort connue, les femmes se rassemblent dans la case de la plus vieille parente du défunt et entament les lamentations $(y \tilde{a} m \tilde{\imath})$. Les musiciens et chanteurs se rendent sur la place pubiique et dans la concession du défunt où ils chantent ses louanges. Les chants des funérailles, au contenu souvent allégorique, varient selon le statut du défunt et selon les liens qui l'unissent à ceux qui évoquent sa mort (I).

Les instruments de musique joués sur la place publique révèlent 1 'appartenance du défunt aux sociétés initiatiques, aux sociétés de culture ou de chasseurs, chacune de ces sociétés se caractérisant par des instruments spécifiques. Un rythme particulier indique à l'auditeur averti qu'il s'agit d'une cérémonie funéraire.

Les instruments joués auprès du corps du défunt sont différents selon qu'il s'agit d'un homme ou d'une femme.

Dans la chambre mortuaire, les hommes âgés ou les vieilles de la familles s'il s'agit de l'inhumation d'une femme, lavent le cadavre à l'eau chaude et au savon, tandis que dans la grande cuisine les vieilles femmes chauffent de l'eau dans trois grands canaris pour une seconde toilette. Celle-ci se déroule près de la grande cuisine, dans un enclos de nattes dressé à cet effet par les jeunes gens du quartier. Le mort $y$ est placé dans la position assise, appuyé contre la gouttière que l'on a enlevée de sa case.

(1) Une femme pleure son mari : "mon Zanga

"il est mort aujourd'hui

"la calebasse de ma jarre s'est brisée

"la perle s'est brisée."

La calebasse qui permet de boire à la jarre d'eau est symbole d'espoir et de vie Cf. Nagognimé Urbain Dembélé : De la tradition orale à la littérature. Etudes Maliennes, $\mathrm{n}^{\circ} 11$, sept.74. 
Le symbolisme de la gouttière est révélé dans les récits sur l'origine de la pluie où l'on dit que le ciel descendit vers la terre et comme une gouttière, apporta l'eau. Le toit en terrasse représente le ciel et la gouttière l'arc-en-ciel qui déversa la pluie sur terre. La gouttière apparaît donc comme un symbole de vie.

Les Minyanka, en effet, considèrent la mort comme une étape à franchir qui aboutit à la résurrection au village des ancêtres. D'autres éléments du rite funéraire précisent cette conception. Ainsi, le cadavre repose toujours la face tournée vers l'est, car l'origine de toute chose se trouve à l'est, la course du soleil d'est en ouest symbolise la création.

Les camarades d'âge du défunt (1) procèdent au lavage rituel dans l'enceinte de nattes. Ils lui denudent les jambes jusqu'aux genoux et le plus âgé les asperge de la main gauche. Tous les hommes présents refont ce geste trois fois.

Ensuite, l'aîné imprègne son index gauche de beurre de karitê qu'il présente aux quatre points cardinaux, d'ouest en est et du nord au sud. Le rituel se trouve ainsi mis en relation avec l'univers. Après avoir posé trois fois les mains sur les genoux du mort, il en frotte les jambes de ce dernier. Les amis et les parents du défunt répètent ces onctions, les hommes trois fois, les femmes quatre fois. Ces nombres symbolisent respectivement le sexe masculin et le sexe féminin.

Pour terminer, les amis feignent de raser la tête du mort à l'aide d'un petit couteau et, s'il s'agit d'une femme, ils défont ses tresses.

Le défunt porte des vêtements blancs enfilés à l'envers, car, dit-on, "la mort est le contraire de la vie".

(1) C'est-à-dire tous ceux qui ont subi la circoncision en même temps que lui et qui ont, plus tard, travaillé ensemble dans la même association de culture. 
Dans tous les cultes où une communication s'établit avec les ancêtres, les acteurs principaux se déplacent à reculons.

Si le défunt est un homme, sa tenue se complète d'un bonnet de coton blanc, dont les pans cachent la figure et la nuque, et d'un bracelet de coton, protection utilisée autrefois pour tirer à l'arc.

Ce bracelet symbolisant la guerre est passé au poignet du défunt, car un homme nè doit pas mourir sans avoir fait la guerre.

Pendant qu'au village, parents et amis s'affairent autour du corps, des jeunes gens n'appartenant pas à la famille, se rendent au cimetière, accompagnés d'un camarade du défunt. Ce dernier assome un poulet noir, figurant la mort, et trace ensuite le plan de la tombe (fanga). Avant d'être sacrifié et consommé par les fossoyeurs, le poulet doit être assommé "car le mort n'a pas été tué" (c'est-à-dire n'a pas subi une mort violente).

La tombe sera creusée différemment selon le statut du défunt et les moyens de sa famille. Tous les parents doivent contribuer au paiement des. fossoyeurs, mais ils présentent ces dons comme "le prix du tabac et de la boisson" du mort.

Dans le cas de mort violente, on dépose le corps dans une simple fosse. A l'est du Minyankala, un type de sépulture appelé "trou de la hyène" (car elle rappelle, dit-on, le terrier de cet animal) est réservé aux femmes: les fossoyeurs creusent une première fosse rectangulaire à partir de laquelle, rëpartis en trois équipes, ils creusent une paroi latérale pour former une petite chambre.

Dans les autres sépultures le corps est déposé au croisement de deux ou trois galeries souterraines : les deux premières se situent dans le même axe, que la troisième rejoint à angle droit. Le croisement évoque l'idée de l'univers. Leur travail terminé, les fossoyeurs se lavent, pour se purifier, avant de rentrer au village où le chef de famille du mort remet à leur aîné sept cauris et $1 \mathrm{FF}$. Ce don permet d'acheter les noix de kola distribuées aux travailleurs. 
La suite du rituel funéraire se déroule à la grande porte, où le corps du défunt recouvert de couvertures blanches est posé sur un brancard confectionné par ses camarades d'âge.

L'aîné des neveux utêrins (1) ou un proche désigné par le conseil de famille s'assied à la tête du mort, sur un mortier renversé; chacun lui offre des cauris. Le fils aînê du défunt, ou tous ses fils si la famille peut se le permettre, offre en sacrifice une chèvre ou un bouc.

Ces sacrifices revêtent plus ou moins d'ampleur selon la richesse de la famille, mais aussi selon les régions. Dans le Nord, par exemple, on n'immole qu'une seule victime.

L'animal donné par le fils aîné est attaché aux pieds du cadavre et sera égorgé au cimetière des enfants.

Le veuf(ve) entouré d'ami(e)s ayant également perdu leur conjoint, quitte la case mortuaire. Examinons ici les funérailles d'un homme. La veuve, tenant en main un épi de gros mil, apparaît la tête rasée et le front ceint d'une bande de coton blanc. Le blarc évoque l'idée de clarté, de bonheur. La veuve en portant ce bandeau espère que son mari l'aidera à avoir le front clair comme l'étoffe et à être heureuse. Les cheveux de la veuve sont déposés dans la poche des vêtements du mort.

Une veuve portant dans un éclat de calebasse de l'eau de la toilette funéraire suit l'épouse du défunt et asperge ses traces de pas afin d'écarter, pour les autres femmes, le risque de perare leur mari. (Le veuf porte un large chapeau de pasteur peul, signe de la fraicheur qu'il demande à son épouse).

Dès l'arrivée du conjoint, on fixe le corps du défunt sur le brancard à l'aide d'une vieille corde à puiser, en ayant soin de laisser libre une main - la gauche pour un homme et la droite pour une femme -, à laquelle on attache un poussin vivant. Avant le départ pour le cimetière, un proche du défunt demande à ceux envers qui le mort était redevable, ainsi qu'a ceux qui lui doivent quelque chose de se manifester.

Dans d'autres villages, ces règlements de dettes se font au cimetière des enfants.

(1) Les neveux utérins (nazo) jouent un rơle de médiateur et exécutent les sacrifices annuels aux ancêtres. 
Les amis du défunt brandissent ensuite le brancard et lui imprime par trois ou quatre fois - selon qu'il s'agit d'un homme ou d'une femre décédée - un mouvement de va et vient face à la grande porte. Cette oscillation rappelle le mouvement de la créa-

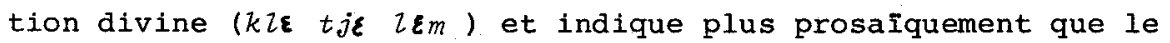
défunt a une nombreuse progéniture. Dans la région de San, le brancard est d'ailleurs balancé au-dessus d'un fils et d'une fille du mort.

Simulant le balayage du chemin les jeunes femmes du quartier ouvrent la voie vers le cimetière. Les griots chantent et ceux qui possèdent un fusil tirent.

Les petits enfants du défunt troublent volontairement la procession par des bousculades et des coups de fouet. Ils lancent des détritus sur le mort et lui adressent des insultes. Tanais que tous les hommes et les femmes s'invectivent mutuellement.

Ces manifestations prennent d'autant plus d'ampleur que le défunt est âgé.

Les parents pour faire avancer le cortège funèbre doivent "acheter le mort" à ses petits enfants.

Sur le chemin du cimetière, le kächyo te ge (1), la "terre de la jarre du village", où se trouve le cimetière des enfants, représente une étape importante.

Plusieurs rites se déroulent en ce lieu à l'ombre d'un arbre imposant qui a été préservé lors de l'installation du village : - le groupe de veufs(ves) renouvelle ici l'onction au beurre de karité, mais en frottant la natte

- le récipient contenant le beurre et tout ce qui a servi à la toilette funéraire est détruit

- le poussin, attaché au bras, et la chèvre offerte par le fils aîné, sont sacrifiés sur les jambes du mort

- les familles aisées offrent un boeuf en sacrifice

- le forgeron (2), procède au divorce et libère le veuf(ve) qui rentre au village.

(1) Cf. J.P. Colleyn, Notes sur la pensée religieuse des Minyanka du Mali, Les cultes généraux.

(2) Le forgeron, premier personnage mythique de la création, jouit d'une grande autorité, on le considère comme médiateur. Cependant on lui attribue une certaine ambivalence : il appartient à la fois au monde terrestre, par son activité indispensable à l'agriculture et au monde chtonien, parsa familiarité avec les métaux extraits du ventre de la terre. 
Dès ce moment, le veuf(ve) doit respecter certains interdits. Fendant toute la durée des funérailles, il (elle) ne consommera aucun des mets cuisinés pour la circonstance, une famille étrangère lui prépare ses repas.

La veuve doit demeurer quatre ou sept jours dans sa case et ne peut répondre à quelqu'un qui, du dehors, lui adresse la parole. Le veuf peut se déplacer à l'intêrieur du village, mais tout voyage lui est interdit pendant sept jours.

Selon les croyances minyanka, le double (dya) peut revenir pendant cette période ; un plat de nourriture est d'ailleurs déposé à son intention à la grande cuisine et le veuf(ve) prend certaines précautions pour éviter tout rapport avec le dya :

de nuit, la veuve porte des vêtements masculins; le veuf dort avec son pantalon dont il noue soigneusement la ceinture afin de décourager les approches du double de son conjoint.

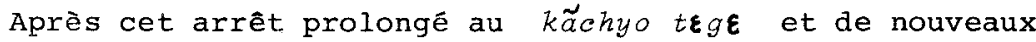
échanges d'injures, le cortège funèbre se dirige vers le cimetière. Arrivé près de la tombe, les porteurs en font plusieurs fois le tour : sept fois pour un homme et huit fois pour une femme. Les parents descendent le corps dans la fosse la plus au nord et l'introduisent dans la logette (l) la face vers l'est.

Le corọs gît sur le côté, un homme repose sur le bras gauche et a la tête au nord tandis qu'une femme repose sur le bras droit. La gauche et la droite signifient respectivement la masculinité et la féminité.

Dans la tombe, différents indices nous rappellent que la mort est une étape avant la vie dans l'au-delà.

Ainsi la face du mort est toujours tournée vers l'est, direction rappelant la création, et les portes du tombeau sont en bois de figuier (toro) qui symbolise la résurrection. Ces portes sont décorées de motifs géométriques noirs, rouges et blancs, identiques à ceux qui figurent sur le sanctuaire du nya.

(1) La logette a la longueur du corps et une largeur et une hauteur d'environ soixante centimètres. Pour les chefs importants, le caveau peut avoir les dimensions d'une chambre. 
Les souterrains et fosses sont combles et un monticule signale 1 'emplacement de la tombe.

Dans 1 'Est du Minyankala la gouttière de la case du défunt est déposée sur ce monticule.

Les parents jettent sur la tombe argent et cauris, dont s'emparent les petits enfants du défunt (dans l'arrondissement de Kimparana) ou les forgerons et les griots (à Somasso).

Les couvertures et de nombreux cauris peuvent être enterrés avec le mort, mais les parents n' acquerront ce droit qu'en dédommageant les petits enfants.

Après avoir fait le tour de la tombe, trois fois pour un mort (ou quatre fois pour une morte), ils rentrent au village accompagnés par les griots.

S'il s'agit des funérailles d'un vieillard, les festivités peuvent se prolonger pendant trois jours, au cours desquels chacun devra se signaler par des dons.

Les griots, principaux béneficiaires, commentent publiquement ; ces largesses.

Les cérémonies funéraires se cloturent par un ou plusieurs repas rituels sans lesquels le defunt ne peut rejoindre le "vil-

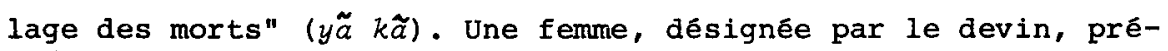
pare, dans la grande cuisine, avec l'aide des filles non mariées du quartier, différents mets.

Ceux-ci mélangés dans un plat en bois sont symboliquement offerts au mort et consommés par les filles et les garçons non circoncis.

A Somasso, un rite semblable est effectué par les filles mariées du defunt. Elles se rendent dans toutes les familles pour se procurer des denrées et proclament qu'elles cherchent leur mort; s'il s'agit de leur père, l'une d'elles se daguise en homme.

Elles deposent un plat dans la grande cuisine en l'honneur du mort et consomment le reste de la nourriture. Ensuite elles brisent et jettent tout ce qui a servi à sa préparation au kãchyot\&ge ("terre de la jarre du village"). 
Si le dśfunt était membre de la société initiatique du kori (1) les danseurs du kori, armés de torches se manifestent la nuit au cours d'un repas rituel.

Ce repas est semblable à celui qui se déroule aux cérémonies annuelles du kori au cours desquelles les initiés consomment des beignets de haricot et se livrent à des combats de torche et de fouet. Dans le carare du kori ces luttes et ce repas symbolisent 1 'accès à la connaissance.

Les initiés volent tout ce qui leur est nécessaire pour cette cérémonie. Le thème du vol de nourriture se retrouve dans un récit sur l'origine de la mort :

"Autrefois les hommes vieillissaient et disparaissaient. Dieu créa la Mort et l'installa en brousse où elle tua des animaux. Un homme trouva ce gibier et demanda à pouvoir le consommer. Mais la Mort ne répondit pas, alors il vola la viande et s'enfuit vers son village.

La Mort armée d'une barre de fer le poursuivit, elle pénétra ainsi au village des hommes et $y$ resta".

Dans l'Est du Minyankala, les funérailles se déroulent en deux temps. La deuxième partie tyєre, (calebasse) se déroule dès que la famille peut faire face aux dépenses somptuaires.

Le tyєrદ répète les rites postérieurs à l'ensevelissement avec beaucoup plus d'ampleur.

$\mathrm{Au}$ terme de cette description, il convient de-mentionner certains rites relatifs à des catégories particulières de décès. La mort peut etre la manifestation de la volonté de Dieu ou d'une divinité, qui sera révêlée par la victime ou le devin ou encore par les circonstances (noyades, foudroiement).

Les funérailles, dans ce cas, se déroulent sans aucun faste et les vêtements du défunt son: déposés ur le toit du sanctuaire de la divinitê qui l'a tué.

La mise en terre du corps de certaines personnes, comme les femmes stériles ou les albinos, peut entraîner la sécheresse.

(1) Cf. J.P. Colleyn, ibid. 
Le corps de la femme sterile, consideré comme un danger pour la fertilite du sol, est place dans une ruche que l'on accroche, de nuit, dans un arbre du village voisin. Ce village se debarassera à son tour de ce fardeau en le plaçant dans un autre village. La ruche sera ainsi déplacée jusqu'à ce qu'elle soit prêcipitée dans un cours d'eau profond.

Le corps de l'albinos, par contre, ne peut etre jeté a l'eau si ce n'est dans le fleuve Niger. Pour préserver la terre du nyama trop puissant de l'albinos, on le deposera de nuit dans une termitière.

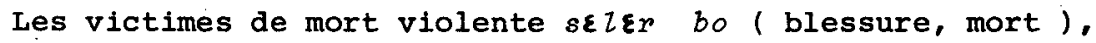
n'atteignent jamais le statut d'ancêtre et errent sur les lieux de $l$ 'accident. Sont considerées comme $s \varepsilon z r$ bo les personnes tuées accidentellement ou volontairement et les suicidés. Les fossoyeurs, tournant le dos au cadavre déshabillé, le poussent des talons dans une. fosse, sans aucune cérémonie. Si les dépouilles de ces morts particuliers sont maniées avec précaution, voire avec crainte, certains cependant en prélèvent des parcelles, car elles interviennent dans la constitution des autels portatifs des sociétés initiatiques.

Dans le cadre de ces notes sur les rites fúnéraires, nous tenonsi à mentionner le simulacre de funérailles qui institue la sacralité du pouvoir du chef de village. Celui-ci peut cependant exercer ses fonctions pendant plusieurs années avant que la cérémonie ait lieu.

Ce rituel d'intronisation n'est pratique que dans la partie nord du Minyankala, dans des villages (1) qui exerçaient autrefois leur domination sur deux ou trois villages environnants. Leur suprématie se traduisait par l'obligation, pour les villages soumis, de payer chacun, chaque année, cinq mille cauris, un mouton, le "prix de la boisson" et de fournir des guerriers, en echange de quoi le village dominant offrait sa protection. Ces chefferies éphèmères se maintenaient tant que le rapport de forces jouait en leur faveur.

(1) Actuellement, cette cérémonie se déroule dans les villages de Kadiala, Kamona, Kéméni, Dougoulo, Bokoni, Yedala, Somasso et dans les arrondissements de Kimparana avec quelques variantes. 
L'essentiel de nos informations ont été recueillies à Somasso, gráce à la collaboration de Kalifa Dao, successeur du chef actuel, dont l'intronisation a eu lieu le 3 janvier et le 21 février 1975.

La cérémonie se déroule en deux temps. La première partie est appelée kulufolı tønge (chef de village, asseoir), ce qui signifie asseoir le chef, c'est à dire l'asseoir dans ses fonctions de chef politique et religieux (I). Au cours d'un simulacre de funérailles, le chef est coiffé d'un bonnet, insigne de son pouvoir.

La deuxième cérémonie gnõtõ for to (bonnet, sortir, dehors) consiste en une présentation du chef au public; elle se déroule après une réclusion de quarante deux jours (2), assimilée à une mort. Le kulufolo doit nécessairement être le descendant direct de l'ancêtre fondateur et être de père et de mère minyanka pour porter le bonnet. Les louanges soulignent sa pureté : il ne peut être d'origine peule ni descendant d'esclaves, ni né après la mort de son père.

De nuit, les chefs de quartiers, ainés des familles fondatrices, emmènent le kulufol, sur la place du village où il s'assied sur une natte face à l'est, appuyé contre une pierre ferrugineuse qui marque ce lieu sacré depuis la création du village. Les griots récitent les louanges du kulufつlo, énumèrent sa généalogie et rappellent les migrations du Mandé.

Les anciens accompagnent ensuite le chef à la grande cuisine pour la toilette mortuaire, lui enfilent les vêtements blancs des morts et le ramènent sur la place publique pour la prise du bonnet. L'épouse du kulufวlつ prend place à ses côtés, entièrement recouverte de couvertures blanches. Désormais, elle est considérée comme morte et son mari renonce à tout rapport sexuel.

(1) Alors que dans la plupart des villages minyanka, la chefferie politique est distincte de la chefferie religieuse, le kulu f $>$ ls "porteur de bonnet" assume ces deux fonctions à la fois.

(2) Soit sept marchés traditionnels après la pose du bonnet. Avant l'ère coloniale, les marchés avaient lieu tous les six jours. Les sages tiennent encore aujourd'hui un calendrier de ces marchés, car ils ponctuent la vie religieuse. 
Seuls les Keita connaissent les paroles à prononcer sur le bonnet et toutes les chefferies font appel à eux pour les rites d'intronisation. A Somasso, la famille Keita, qui procède

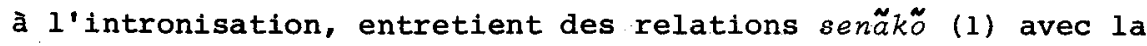
famille cheffale des Dao.

L'ainé des Keita pose le bonnet sacré (gnötõ ni), bonnet rond de coton blanc (dans l'arrondissement de Kimparana le bonnet a deux ailerons descendant sur les oreilles), sur la tête du chef en murmurant les paroles secrètes, il proclame ensuite à haute voix : "Tout le village t'a choisi et te donne ce bonnet pour être son chef".

A $l^{\prime \prime a u b e ~ l e ~ k u l u f s l o ~ e s t ~ r e c o n d u i t ~ c h e z ~ l u i ; ~ d e ́ s o r m a i s ~ i l ~ e s t ~}$ considéré comme un ancêtre, il est sacré et représente le village. Il perd son nom, il ne peut plus y répondre sans risquer la mort, on doit l'appeler kulufs lo tangbula (2).

Pendant les quarante deux jours de réclusion, il est considêré comme mort, il ne peut sortir de chez lui, il ne peut répondre à quiconque lui parlerait du dehors.

La cérémonie de sortie se fait avec le plus de faste possible, tous les membres des villages reconnaissant I'autorité du ku uf contribuent au paiement de ses nombreux boubous at participent aux frais occasionnés par l'intronisation.

Le chef, toujours vêtu de blanc, sort de sa case, et apparaît précédé d'un joueur de trompe, évoluant en marche arrière (3) et suivi d'un vieux portant sa houe.

La trompe, constituée d'une défense d'éléphant recouverte de peau de vache et ornée d'une figurine humaine en bois, représente le voix du kulufols. Cette figurine évoquerait le premier Minyanka tué par les Français lors de la conquête coloniale.

Le chef, tête baissée, et sa suite se dirige à petits pas rythmés par les tambours et les chants des griots, vers la grande porte, où la foule vient grossir le cortège qui contourne alors le village.

(1) Relation senäkõ qui se traduit par une alliance, une assistance mutuelle, la prohibition du mariage entre les deux familles, des relations à plaisanteries et le vol réciproque.

(2) Aucun informateur n'a pu nous donner la traduction littérale de ce terme, qui évoque l'idée de noblesse, de purețé et de bonheur.

(3) Le kulufs 2 s appartient déjà au monde des morts. 
Sur le passage de la procession, une femme s'affaire auprès d'un puits pour signifier que la vie suit son cours.

La suite de la cérémonie se déroule au kãchyo trge oũ un enclos de nattes est dressé et protégé par de nombreux hommes en armes. Le kulufolo prend place dans cet abri et à ses côtés son épouse, toujours enveloppée d'un linceul. L'al̂né des Keita aide le chef à endosser, par dessus ses vetements blancs, un grand boubou tissé à petits carreaux noirs et blancs et le coiffe, par dessus son bonnet blanc, d'un second bonnet, semblable au premier mais de couleur rouge.

Pendant toute la journée le kulufวlつ tangbula reste assis dans l'enclos, sur la natte. A ses côtés sont déposés une canne en forme de serpent, en bois de figuier (toro) et un panier dans lequel les visiteurs qui défilent déposent argent et cauris.

Tandis que chacun présente ses salutations au chef, les Keita volent tout ce qu'ils trouvent dans le village.

Après la cérémonie du gnõtõ forolo le chef peut sortir de sa case mais il doit rester sur le territoire du village et toujours se déplacer en compagnie de deux jeunes gens armés, chargés de défendre le bonnet, insigne du pouvoir.

Autrefois, un village adverse pouvait s'emparer du bonnet sacré, c'était là le signal du déclenchement des hostilités entre les deux villages. Si le village ayant perdu le bonnet ne parvenait pas à le récupérer, il était soumis et perdait le droit de porter cet insigne.

Toujours présent au village, le chef doit être mis au courant de tout, il tranche les litiges de façon irrévocable, tout qui le contredit risque la mort. Comme chef religieux, il préside à tous les sacrifices collectifs du village.

Le kulu fo ls tangbula ne travaille plus, la communaúté villageoise l'entretient : chacun doit lui donner une part de tout ce qu'il a cultivé (1) et personne ne peut le sáluer les mains vides.

(1) Un panier de plus ou moins $20 \mathrm{~kg}$ par récolte et par familile. 


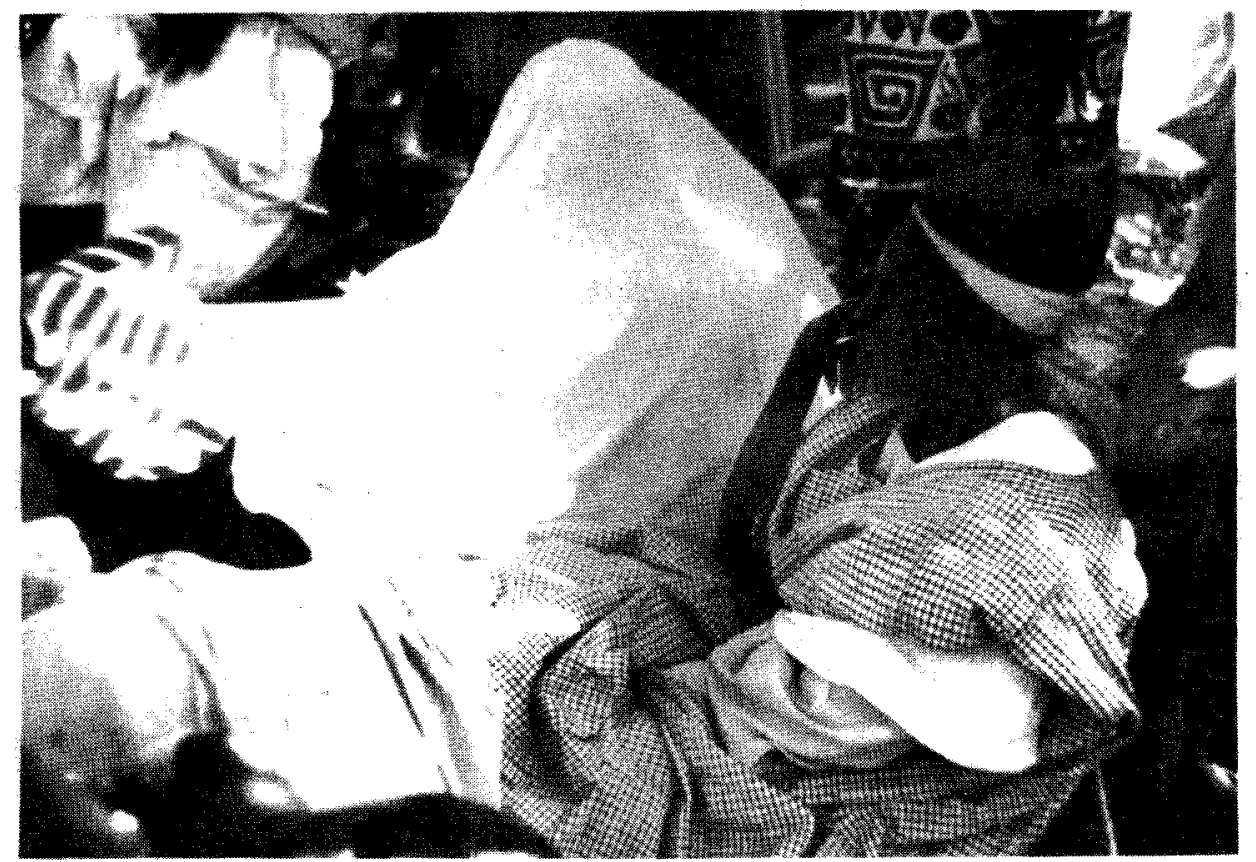

Chef du village de Somasso (Mali) lors de la cérémonie d'intronisation. A ses côtês, son épouse enveloppée d'un linceul. ( photo Philippe Jespers ) 
Nous terminerons par trots extraits (1) des chants d'intronisation; ils soulignent la pureté du chef et rappellent sa fonction de juge suprême, le caractère sacré de son pouvoir qui s'appuie sur les puissances qui consacrent la fondation du village.

"Que dieu (klE) t'aide

"Que la vieille porte du village t'aide

"Que le bois sacré du village t'aide

"Que la jarre du village t'aide (2)

"Que le dasiri du village t'aide (2)

"Premier fils, chef de village

"Que tu entendes cela

"Ils t'ont fait tangbuza

"Tu es pur, pur

"Tu arrives à la fin de toutes choses

"Dieu a fait ton nom semblable à lui

"Oh notre premier fils de dieu

"Tu es le bras en train de descendre, sévèrement".

(1) Enregistrés par P. Jespers à Somasso, lors du gnötô farozo. (2) J.P. Colleyn, ibid. 